\title{
50 Jahre Kleihauer-Betke-Test: Eine vergessene Methode?
}

\section{Years Kleihauer-Betke Test: A Forgotten Method?}

Vor 50 Jahren wurde die Methode zum Nachweis fetaler Erythrozyten in Blutausstrichen erstmals von Kleihauer, Braun und Betke beschrieben [5]. Die fundamentale Tragweite der Mitteilung für die Entwicklung in vielen medizinischen Subdisziplinen, insbesondere auch in der perinatalen Medizin, wird in dem Artikel von Frau Prof. Dr. E. Kohne gewürdigt [6].

Aus der Sicht der modernen Perinatologie ist diese Methode nicht aus den Laboranalysen wegzudenken, obschon sie unter den Bedingungen moderner Großlaboratorien an Klinika der Maximalversorgung zunehmend in Vergessenheit gerät. Die erneut zunehmende Zahl von Früh- und Neugeborenen mit Rhesusinkompatibilität und deren gravierende Konsequenzen ist in diesem Kontext zu erwähnen [4]. Ursache hierfür ist neben der unterlassenen Vorsorge die mangelhafte Überprüfung der Effizienz der Anti-D-Prophylaxe durch Unterlassen eines Kleihauer-Betke-Testes im Anschluss an die Applikation von Anti-D-Immunglobulin. Kontroversen um die notwendige Dosis von Anti-D sind ohne entsprechende Erfolgskontrollen sinnlos [2].

Darüber hinaus ist die Diagnose des fetomaternalen Transfusionssyndroms als eine der häufigsten Ursachen neonataler Anämien zunehmend in Vergessenheit geraten [7]. Auch hier ist der Kleihauer-Betke-Test die Basis für die Diagnostik und sollte als Standard wie bei der zuvor erwähnten Problematik jederzeit in Perinatalzentren verfügbar sein.

Die genannten Beispiele mögen belegen, dass gerade unter den Bedingungen einer hoch spezialisierten Perinatologie mit entsprechender pränataler Diagnostik und sich rasant verbessernden
Behandlungsergebnissen, alte diagnostische Standards einen weiterhin unverzichtbaren Stellenwert haben und diese nicht durch die reduzierte Häufigkeit von schweren symptomatischen RhInkompatibilitäten und anderen Komplikationen in Vergessenheit geraten dürfen [3]. Gleiches gilt für die Diagnostik von Hämoglobinopathien [1]. Insofern ist die Anerkennung der wissenschaftlichen Leistung nicht nur in die Vergangenheit gerichtet, sondern auch unverzichtbarer Bestandteil zeitgemäßer diagnostischer Methoden in einer hochdifferenzierten Perinatologie.

L. Gortner

\section{Literatur}

1 Hartmann K, Kulozik AE. Genetische modulierende Faktoren der Klinik der homozygoten Sichelzellerkrankung. Klin Pädiatr 2006; 218: 170-173

2 Howard HL, Martlew VJ, MacFadyen IR, Clarke CA. Preventing Rhesus D haemolytic disease of the newborn by giving anti-D immunoglobulin: are the guidelines being adequately followed? $\mathrm{Br} \mathrm{J}$ Obstet Gynaecol 1997; 104: 37-41

3 Katiyar R, Kriplani A, Agarwal N, Bhatla N, Kabra M. Detection of fetomaternal hemorrhage following chorionic villus sampling by Kleihauer Betke test and rise in maternal serum alpha feto protein. Prenat. Diagn 2007; 27: 139-142

4 Kenneth J, Moise JR. Management of Rhesus Alloimmunization in Pregnancy. Obstetrics \& Gynecology 2002; 100: 600-611

5 Kleihauer E, Braun H, Betke K. Demonstration von fetalem Blut in den Erythrozyten eines Blutausstriches. Klin Wschr 1957; 35: 637-638

6 Kohne E. 50 Years Kleihauer-Betke Test. Klin Pädiatr 2007; 219: 252-253

7 Salim R, Ben-Shlomo I, Nachum Z, Mader R, Shalev E. The incidence of large fetomaternal hemorrhage and the Kleihauer-Betke test. Obstet Gynecol 2005; 105: 1039-1044
Bibliografie

DOI 10.1055/s-2007-985149

Klin Pädiatr 2007; 219: 251

(c) Georg Thieme Verlag KG

Stuttgart · New York

ISSN 0300-8630

Korrespondenzadresse

Prof. Dr. Ludwig Gortner

Kliniken für Kinder- und

Jugendmedizin

Universitätsklinikum des

Saarlandes

66421 Homburg/Saar

ludwig.gortner@uks.eu 